\title{
The Changing Face of Primary Care Research and Practice-Based Research Networks (PBRNs) in Light of the COVID-19 Pandemic
}

\author{
Hazel Tapp, BSc, PhD
}

The COVID-19 outbreak is a stark reminder of the ongoing challenge of emerging and reemerging disease, the human cost of pandemics and the need for robust research. ${ }^{1}$ For primary care, the advent of COVID-19 has forced an unprecedented wave of practice change. In turn, Practice-Based Research Networks (PBRNs) must rapidly pivot to address the changing environment and the critical challenges faced by primary care. The pandemic has also impacted the ability of PBRNs to deploy traditional research methods such as face-to-face patient and provider interactions, practice facilitation, and stakeholder engagement. Providers need more relevant, patient-centered evidence and the skills to effect change. These skills will become more important than ever as primary care practices evolve in response to the current COVID-19 pandemic and the disparities in health outcomes highlighted by COVID-19 and the global Black Lives Matter social movement for justice. Throughout this issue, authors detail the work conducted by PBRNs that demonstrate many of these evolving concepts. Articles explore how PBRNs can evaluate COVID-19 in primary care, the role of PBRNs in quality improvement, stakeholder engagement, prevention and chronic care management, and patient safety in primary care. $(\mathrm{J}$ Am Board Fam Med 2020;33:645-649.)

The COVID-19 outbreak is a stark reminder of the ongoing challenge of emerging and reemerging disease, the human cost of pandemics and the need for robust research. ${ }^{1}$ For primary care, the advent of COVID-19 has forced an unprecedented wave of practice change. In turn, Practice-Based Research Networks (PBRNs) must rapidly pivot to address the changing environment and the critical challenges faced by primary care. The pandemic has also impacted the ability of PBRNs to deploy traditional research methods such as face-to-face patient and provider interactions, practice facilitation, and stakeholder engagement. Key new areas for evaluation are the move to virtual visits, the health impact of COVID on practice staff, providers and patients (including racial disparities in outcomes), and practice business functions.

From the Department of Family Medicine, Atrium Health, Charlotte, NC.

Funding: Funding for this work was supported by Atrium Health.

Conflict of interest: The author has no conflicts of interest.

Corresponding author: Hazel Tapp, BSc, PhD, Atrium

Health, 2001 Vail Av, Charlotte, NC 28269 (E-mail: hazel.tapp@atriumhealth.org).
PBRNs have traditionally played a role in translation of research into practice, and more recently in the implementation and evaluation of quality improvement efforts. ${ }^{2-9}$ Since 2000, when Congress asked the Agency for Health care Research and Quality to identify and support primary care research, ${ }^{10}$ PBRNs have become a resource for accelerating the translation of research into practice with research generated by PBRNs shown to improve outcomes for patients and practices. ${ }^{11}$ PBRNs can also be thought of as able to help identify problems in daily practice, demonstrate whether treatments are effective and sustainable in realworld settings, and provide the "laboratory" for testing system improvements. ${ }^{12}$

Primary care physicians are more motivated than ever to incorporate evidence-informed decision making into everyday practice particularly in combination with patient engagement techniques such as shared decision making. ${ }^{13}$ Unfortunately, physicians are still hampered by the need for more relevant, patient-centered evidence and the need to acquire the skills to effect change. PBRNs incorporate community-engaged research, participatory implementation research, quality improvement and implementation science initiatives, continuing 
education, and the training of future generations of translational investigators. ${ }^{14}$ PBRNs use quality improvement and implementation science to inform and quantify improvements. In addition, sustained collaborations with providers, patients, and other relevant stakeholders such as local and national advocacy groups and community-based organizations are used to enhance the work of PBRNs. ${ }^{15}$

Currently, quality improvement and implementation science knowledge are still predominantly in the domain of researchers within PBRNs. These skills need to be translated to user-friendly tools that are accessible and readily used by health care practitioners. ${ }^{16}$ Integration of new skills into practice is not easy because it often requires new kinds of relationships, conceptual frameworks, and even languages for clinicians, patients, researchers, academic institutions, and funding agencies. An emerging role for PBRNs is both traditional primary care research and collaborative learning communities that can identify, disseminate, and integrate new knowledge. ${ }^{9}$ These skills will become more important than ever as primary care practices evolve in response to the current COVID-19 pandemic and the disparities in health outcomes highlighted by COVID-19 and the global Black Lives Matter social movement for justice.

Throughout this issue, authors detail the work conducted by PBRNs, which demonstrate many of these evolving concepts. Articles explore how PBRNs can evaluate COVID-19 in primary care, the role of PBRNs in quality improvement, stakeholder engagement, prevention and chronic care management, and patient safety in primary care.

PBRNs are critical laboratories for studying the implementation of evidence-based practices in realworld settings. DeVoe et a ${ }^{17}$ describe how the current pandemic and primary care's response to it are among the most impactful natural experiments in our lifetime, presenting an opportunity to demonstrate PBRNs' power and value in supporting dissemination and implementation science. The collaboration between a community health care center PBRN and implementation scientists are being leveraged to evaluate how community health care centers across the country are responding to the COVID-19 pandemic. Researchers will use medical record data, telemedicine trainings, and qualitative interviews with practices over a 12 -month period to identify practice adaptation to delivery of care.
Two articles illustrate how PBRNs facilitate advancements in quality improvement. Project ECHO (Extension for Community Health care Outcomes) uses case-based telementoring to support community clinicians to deliver best-practice care. ${ }^{18}$ McDonnell Elder et al ${ }^{19}$ describe how 1 PBRN has created a statewide network for ECHO programs. The PBRN facilitated a unique funding stream for the ECHO programs by partnering with payers and health care systems. Using examples of tobacco cessation, chronic pain and opioid prescribing, and diabetes management, the authors describe how the collaboration enhanced practice recruitment and retention and improve financial stability. Practice facilitation is an effective approach to implementing quality improvement in primary care. Regular facilitator-practice interactions are necessary for successful facilitation. Ye et $\mathrm{al}^{20}$ sought to identify practices facilitation barriers using a time series analysis to evaluate facilitation activities across multiple practices. While most facilitation activities occurred at regular practicespecific tempos, nearly all practices experienced at least 1 delay with facilitation, ultimately showing that number of facilitation delays correlated with lower intervention completion.

PBRNs frequently engage community members and clinic staff through community advisory boards and patient advisory councils. ${ }^{21}$ Additional strategies such as virtual solutions are now needed as PBRNs consider how to facilitate longitudinal engagement of stakeholders and the needs for virtual engagement created by COVID-19. Engster et $\mathrm{al}^{22}$ describe the creation of a virtual Parent Panel to engage parents remotely and use their input for child health research. The authors utilized an existing research study on pain management during routine child vaccinations to develop regular communication with a group of parents/patient stakeholders to provide feedback on research ideas. Although not meeting the definition of full community-based participatory research, given the large geographic area the PBRN serves, and barriers associated with bringing together in-person meetings, this strategy represents a pragmatic move forward in patient engagement, particularly in the light of COVID-19.

Additional approaches to patient engagement were described by Dickinson et $\mathrm{al},{ }^{23}$ where practice facilitation combined with patient engagement enhanced implementation of new models of care. Mungia et $\mathrm{al}^{24}$ discuss 7 years of dental practitioner 
engagement activities within a national dental PBRN. This network used a broad range of activities to engage community practitioners showing success in sustaining a high level of practitioner engagement in research that was relevant to everyday clinical practice. Nagykaldi et $\mathrm{al}^{25}$ describe using stakeholder partnerships to show community-wide health improvement across a rural PBRN. The Healthier Together study aimed to implement and evaluate a sustainable, communitybased preventative care patient outreach model. Stakeholders included community-based wellness coordinators, primary care providers, county health departments, local hospitals, and health information exchange networks.

Prevention and chronic disease management is a large part of the work of primary care. Support and evaluation of prevention and chronic disease by PBRNs offers robust evaluation of current practice, new innovations and best practices. ${ }^{26,27}$ Krist et $\mathrm{al}^{28}$ point out that while barriers to preventive screening, such as colon cancer screening, are well documented, less is known about the relative importance of patient, clinician, health system, and communication factors associated with recommended screening, particularly for vulnerable populations. Based on the results of a large patient survey, the authors were able to show that having a long-term relationship with a primary care clinician and sharing decisions are key drivers to ensure evidence-based preventive care for underserved populations. In another study focusing on vulnerable populations, Heintzman et $\mathrm{al}^{29}$ examined potential disparities in care provided to Latino children compared with non-Hispanic white children and showed that in this multistate network of clinics, Latino children were less likely to have their asthma entered on their problem list than non-Hispanic white children, but otherwise did not receive inferior care. The authors suggest that asthma disparities experienced by this population may occur at other stages of care such as initial diagnosis or not actually filling the prescription medication filling, rather than at the stage of appropriate prescribing. Kwan et $\mathrm{al}^{30}$ used mixedmethods qualitative research to compare different models of diabetes shared medical visits. Researchers used surveys, interviews, and observation to assess practice contextual factors, such as practice size, location, payer mix, change and work culture, motivation to participate, and clinical and administrative capacity. Registries are foundational elements for the Chronic Care Model and the Patient-Centered Medical
Home. $^{31}$ Previous research has demonstrated that registries are effective for improving clinical guideline adherence for care of patients with type 2 diabetes. However, registry implementation has typically relied on intensive support (such as practice facilitators) for practice change and care improvement. Sabo et $\mathrm{al}^{32}$ showed that a remotely delivered, low-intensity peer mentoring intervention can support the use of diabetes registries in primary care, reducing the intensity of support needed. Primary care obesity management has many barriers for providers including the reality of managing patients' expectations. Brooks et $\mathrm{al}^{33}$ used a qualitative approach to discover what motivated primary care clinicians to take part in an obesity research intervention. Providers felt that the research project provided a concrete plan to address their ongoing clinical care need for effective obesity treatment and management, offered help to frustrated physicians who felt a deep professional duty to care for all their patients' problems, and because it demonstrated their commitment to improving the health of the broader community. Team-based approaches to disease management continue to be important areas of study. Clinical pharmacists provide important services in patient care and have the time to devote to more granular dietary and medical management. Norton et $\mathrm{al}^{34}$ showed that collaboration of pharmacists and physicians in the primary care setting is associated with improved diabetes outcomes and substantially reduces costs.

Patient safety in primary care is a priority. Issues such as overprescribing medications, diagnoses, transitions, referrals, and inappropriate testing are emerging areas of concern. ${ }^{35}$ Lai et $\mathrm{al}^{36}$ discovered that providers and patients prefer function-based conceptualizations such as not causing harm and viewing patients' needs holistically, that better reflect front line personnel and patients' experiences rather than domain-based conceptualizations, such as overprescribing medications, which are favored by experts.

Going forward, the physical, psychological, and societal consequences of COVID-19 will need to be considered in all aspects of primary care and associated practice-based research needs.

The author would like to acknowledge the assistance of Sylvia Tapp in the preparation of this manuscript.

To see this article online, please go to: http://jabfm.org/content/ 33/5/645.full. 


\section{References}

1. Fauci AS, Lane HC, Redfield RR. Covid-19Navigating the uncharted. N Engl J Med 2020;382: 1268-9.

2. Mays GP, Hogg RA, Castellanos-Cruz DM, Hoover AG, Fowler LC. Public health research implementation and translation: evidence from practice-based research networks. Am J Prevent Med 2013;45:752-62.

3. Nagykaldi Z. Practice-based research networks at the crossroads of research translation. J Am Board Fam Med 2014;27:725-9.

4. Tapp H, Dulin M. The science of primary healthcare improvement: potential and use of community-based participatory research by practicebased research networks for translation of research into practice. Exp Biol Med (Maywood) 2010; 235:290-9.

5. Fagnan LJ, Simpson MJ, Daly JM, et al. Adapting boot camp translation methods to engage clinician/ patient research teams within practice-based research networks: a report from the INSTTEPP Trial and Meta-LARC Consortium. J Patient Cent Res Rev 2018;5:298-303.

6. Haggerty T, Lewis W, Plaugher C, et al. Residents' views on research and quality improvement training can guide practice-based research network collaboration. WV Med J 2018;2018.

7. Goldstein KM, Vogt D, Hamilton A, et al. Practice-based research networks add value to evidence-based quality improvement. Healthc (Amst) 2018;6:128-34.

8. Kairys S, Wasserman R, Pace W. Practice-based quality improvement/research networks: full speed forward. Acad Pediatr 2013;13:S12-S13.

9. Mold JW, Peterson KA. Primary care practicebased research networks: working at the interface between research and quality improvement. Ann Fam Med 2005;3:S12-S20.

10. Tierney WM, Oppenheimer CC, Hudson BL, et al. A national survey of primary care practice-based research networks. Ann Fam Med 2007;5:242-50.

11. Westfall JM, Roper R, Gaglioti A, Nease DE, Jr. Practice-based research networks: strategic opportunities to advance implementation research for health equity. Ethn Dis 2019;29:113-8.

12. Westfall JM, Mold J, Fagnan L. Practice-based research-"Blue highways" on the NIH roadmap. JAMA 2007;297:403-6.

13. Bonner C, Fajardo MA, Doust J, McCaffery K, Trevena L. Implementing cardiovascular disease prevention guidelines to translate evidence-based medicine and shared decision making into general practice: theory-based intervention development, qualitative piloting and quantitative feasibility. Implement Sci 2019;14:86.
14. Riley-Behringer M, Davis MM, Werner JJ, Fagnan LJ, Stange KC. The evolving collaborative relationship between practice-based research networks (PBRNs) and clinical and translational science awardees (CTSAs). J Clin Trans Sci 2017;1:301-9.

15. Gaglioti AH, Walston D, Vasquez Guzman CE, et al. A practical approach to establishing a practice-based research network stakeholder engagement infrastructure. J Am Board Fam Med 2019;32:695-704.

16. Westerlund A, Sundberg L, Nilsen P. Implementation of implementation science knowledge: the research-practice gap paradox. Worldviews Evid Based Nurs 2019;16:332-4.

17. DeVoe JE, Likumahuwa-Ackman SM, Angier HE, et al. A practice-based research network (PBRN) roadmap for evaluating COVID-19 in community health centers: a report from the OCHIN PBRN. J Am Board Fam Med 2020;33:774-778.

18. Furlan AD, Pajer KA, Gardner W, MacLeod B. Project ECHO: building capacity to manage complex conditions in rural, remote and underserved areas. Can J Rural Med 2019;24:115-20.

19. McDonnell MM, Elder NC, Stock R, Wolf M, Steeves-Reece A, Graham T. Project ECHO integrated within the Oregon Rural Practice-based Research Network (ORPRN). J Am Board Fam Med 2020;33:789-795.

20. Ye J, Zhang R, Bannon JE, et al. Identifying practice facilitation delays and barriers in primary care quality improvement: a report from Evidence NOW. J Am Board Fam Med 2020;33:655-664.

21. Westfall JM, VanVorst RF, Main DS, Herbert C. Community-based participatory research in practice-based research networks. Ann Family Med 2006;4:8-14.

22. Engster SA, Fascetti C, Mykita A, Pompa K, Cohen Reis E. Bringing parent voices into a pediatric research network through a virtual parent panel. J Am Board Fam Med 2020;33:665-674.

23. Dickinson WP, Nease DE, Jr, Rhyne RL, et al. Practice transformation support and patient engagement to improve cardiovascular care: from EvidenceNOW Southwest (ENSW). J Am Board Fam Med 2020;33:675-686.

24. Mungia R, Funkhouser E, Makhija SK, et al. Practitioner engagement in activities of the national dental practice-based research network (PBRN): 7Year Results. J Am Board Fam Med 2020;33:687-697.

25. Nagykaldi Z, Scheid D, Zhao YD, Mishra B, Greever-Rice T. A sustainable model for preventive services in rural counties: the Healthier Together study. J Am Board Fam Med 2020;33:698-706.

26. Pinto RM, Park S. COVID-19 pandemic disrupts HIV continuum of care and prevention: implications for research and practice concerning community-based organizations and frontline providers. AIDS Behav 2020 [Epub ahead of print]. 
27. Corvin J, Aguado Loi C, Alfonso M, et al. Translating research into practice: employing community-based mixed methods approaches to address chronic disease and depression among Latinos. J Behav Health Serv Res 2017;44:574-89.

28. Krist AH, Hochheimer CJ, Sabo RT, et al. Patient, clinician, and communication factors associated with colorectal cancer screening. J Am Board Fam Med 2020;33:779-784.

29. Heintzman J, Kaufmann J, Lucas J, et al. Asthma care quality, language, and ethnicity in a multi-state network of low-income children. J Am Board Fam Med 2020;33:707-715.

30. Kwan BM, Rementer J, Richie ND, et al. Adapting diabetes shared medical appointments to fit context for practice-based research (PBR). J Am Board Fam Med 2020;33:716-727.

31. Coleman K, Wagner E, Schaefer J, Reid R. Redefining primary care for the 21 st century. Rockville, MD: Agency for Healthcare Research and Quality; 2016.
32. Sabo RT, Etz RS, Gonzalez MM, et al. Low-intensity intervention supports diabetes registry implementation: a cluster-randomized trial in the Ambulatory Care Outcomes Research Network (ACORN). J Am Board Fam Med 2020;33:728-735.

33. Veazey Brooks J, Kimminau KS, McCrea-Robertson S, Befort C. Rural family medicine clinicians' motivations to participate in a pragmatic obesity trial. J Am Board Fam Med 2020;33:736-744.

34. Norton MC, Haftman ME, Buzzard LN. Impact of physician-pharmacist collaboration on diabetes outcomes and health care use. J Am Board Fam Med 2020;33:745-753.

35. Montano MF, Mehdi H, Nash DB. Annotated bibliography: understanding ambulatory care practices in the context of patient safety and quality improvement. Am J Med Qual 2016;31:29S-43S.

36. Lai AY, Yuan CT, Marsteller JA, et al. Patient safety in primary care: conceptual meanings to patients and the health care team. J Am Board Fam Med 2020;33:754-764. 